\title{
Identification of Pneumonia Based on an Electronic Nose
}

\author{
Shih-Wen Chiu ${ }^{1}$, Hung-Yi Hsieh ${ }^{1}$, Han-Wen Kuo ${ }^{2}$, Shang-Ren Yang ${ }^{1}$, Li-Chun Wang ${ }^{3}$, Kea-Tiong \\ Tang $^{1}$, Chung-Hung Shih ${ }^{4}$ \\ ${ }^{1}$ Department of Electrical Engineering, National Tsing-Hua University, Hsinchu, Taiwan, \\ swchiu@larc.ee.nthu.edu.tw \\ ${ }^{2}$ Department of Chemistry, National Central University, Taoyuan, Taiwan, \\ ${ }^{3}$ Chemical System Research Division, Chung-Shan Institute of Science and Technology, Taoyuan, \\ Taiwan, \\ ${ }^{4}$ School of Respiratory Therapy, Taipei Medical University, No. 250, Wu-Hsing Street, Taipei 110, \\ Taiwan
}

\begin{abstract}
:
Pneumonia is the main reason which causes patients death in hospital. The novelty breath gas analysis method probability offers doctors to identify and monitor this clinical condition immediately. But the gas analysis equipments, such as gas chromatography (GC) and Fourier transform infrared spectroscopy (FTIR), are very accurate but expensive, time consuming, and nonportable. The electronic nose (e-Nose) which has advantages of low cost and easy operation is more suitable to realize an infection pneumonia early alert system for personal use. In this work, we collected the sampling gas from patient who got infection pneumonia and then had been healed respectively. We used the commercial e-nose to get the raw data, and analyzed by principal component analysis (PCA). The results show that we could distinguish between patients with infection pneumonia and patients who had been healed.
\end{abstract}

Key words: breath gas analysis, gas chromatography, Fourier transform infrared spectroscopy, electronic nose, infection pneumonia, principal component analysis

\section{Introduction}

In hospital, when patients finish their treatment, they are usually weak and easy to be infected by bacteria. In this cause, doctors have to spend a plenty of time on diagnosis like chest X-ray and blood analysis. Before the results, the patients delay the treatment. Some patients die in this period. Therefore, the immediately diagnosis is significant. In recent year, smelling breath is one of the methods to identify the infection pneumonia. A reasonable supposition is that the paramedic always smells a special flavor from the patients frequently. The smells have the possibility which is caused by the pneumonia. To detect the specific smell, we could identify the pneumonia without the complex analysis method. However, the traditional gas analysis methods is to bring the sampling gas back to the laboratory, and then process by gas chromatography mass analyzer (GC-MS) or Fourier infrared spectrometer (FT-IR).
Although GC-MS and FTIR are all accurate instruments, they are not suitable for personal health use. These instruments are large-scale, high price, and require timeconsuming expert operation. Therefore, an immediate, reliable, fast, and low cost instrument is very important of the gas analysis for pneumonia identification. Fortunately, the electronic nose (e-nose) offers another choice for the gas identification of disease. The e-nose uses the non-specific sensor array to form a specific response pattern of each specific gas, and identifies this specific pattern for disease identification. Recently, the e-nose has been widespread in medical service application, including skin medicine, lung cancer, the kidney disease, reproduction medicine, diabetes, asthma and so on. Here, we collected the sampling gas from patient who got infection pneumonia and then had been healed respectively. Then, we used the commercial e-nose to get the raw data, and analyzed by principal component analysis (PCA). 


\section{Experimental}

We construct the standard operating procedure (SOP) of breath gas collecting from pneumonia patient. The gas of sample is aspirated from the end of the pipeline by respirator. In order to obtain significant data of sensor response, we choice the commercial machine called "Cyranose 320". Cyranose 320 is a reliable portable electronic nose which is developed by Cyrano Sciences. In our case, Cyranose 320 was just a device for raw data collecting. There are 32 sensor components which could be used for noninvasive medical diagnostics, hazardous materials identification, and food spoilage detection, and many other applications. The 32 sensor components are all resistor type and coated with 8 type of sensing materials. When the test gas flows in, the resistor is changed. Breath samples from each patient is collected with a 500mL gas sampling bag, and then pour into device through a gas pumper at $10 \mathrm{~mL} / \mathrm{s}$. Because the capacity of the sampling bag is $500 \mathrm{~mL}$, the total injection time for one sample is $t=500 / 10=50 \mathrm{~s}$. The resistances of the sensors change from $R_{0}$ to $R_{S}$ when they are exposed to sample gas. The information containing $R_{S}-R_{0} / R_{0}$, which serves as the odor data for further analysis, is obtained. There are
32 components of sensor array, but the weighting of signals do not the same. Principal component analysis (PCA) is a mathematical procedure that uses an orthogonal transformation to convert a set of observations of possibly correlated variables into a set of values of uncorrelated variables called principal components. Principal components are guaranteed to be independent only if the data set is jointly normally distributed. PCA is sensitive to the relative scaling of the original variables.

\section{Results}

Figure 1 shows the PCA result. The patients took X-ray to confirm the restored situation of infecting. We can distinguish the patients who got the infection pneumonia and had been recovered in two groups. However, it is not an eminent boundary, the degree of each restored patients is different. As the result, it may be helpful for doctor to early diagnose the patient who gets the infection pneumonia. Beside, the result suggests that the doctor could judge the degree of the recovery of pneumonia.

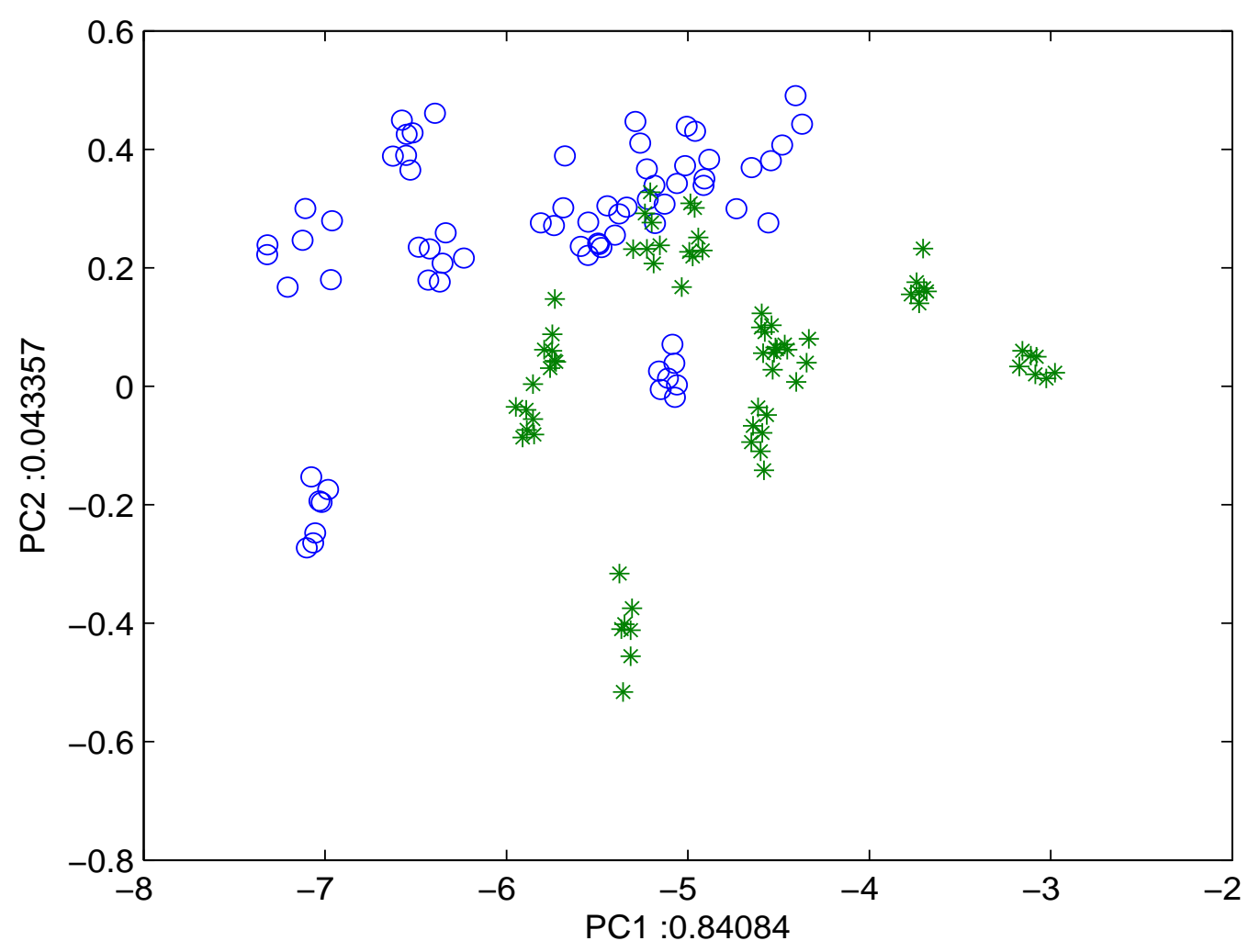

Fig. 1. The PCA result of the patient who got infection pneumonia and then had been healed.. 


\section{References}

[1] D'Amico, A., et al., Identification of melanoma with a gas sensor array. Skin Res Technol, 2008. 14(2): p. 226-236.

[2] Chen, X., et al., A study of the volatile organic compounds exhaled by lung cancer cells in vitro for breath diagnosis. Cancer, 2007. 110(4): p. 835-844.

[3] Phillips, M., Can the Electronic Nose Really Sniff out Lung Cancer? American Journal of Respiratory and Critical Care Medicine, 2005. 172: p. 1060-1061.

[4] Natale, D., et al., Lung cancer identification by the analysis of breath by means of an array of non-selective gas sensors. Biosens Bioelectron, 2003. 18(10): p. 1209-1218.

[5] Voss, A., et al., Smelling renal dysfunction via electronic nose. Annals of biomedical engineering, 2005. 33(5): p. 656-660. 